\title{
Electrospun Chitosan/Polyethylene Oxide Nanofibrous Scaffolds with Potential Antibacterial Wound Dressing Applications
}

\author{
Tony T. Yuan, ${ }^{1,2,3}$ Phillip M. Jenkins, ${ }^{1,2}$ Ann Marie DiGeorge Foushee, \\ Angela R. Jockheck-Clark, ${ }^{1}$ and Jonathan M. Stahl ${ }^{1}$ \\ ${ }^{1}$ Naval Medical Research Unit San Antonio, JBSA, 3650 Chambers Pass, Bldg. 3610, San Antonio, TX 78234, USA \\ ${ }^{2}$ Oak Ridge Institute for Science and Education, 100 ORAU Way, Oak Ridge, TN 37830, USA \\ ${ }^{3}$ Department of Biomedical Engineering, University of Texas at San Antonio, One UTSA Circle, San Antonio, TX 78249, USA \\ Correspondence should be addressed to Jonathan M. Stahl; jonathan.m.stahl.mil@mail.mil
}

Received 15 June 2016; Revised 13 September 2016; Accepted 22 September 2016

Academic Editor: Ilaria Armentano

Copyright (C) 2016 Tony T. Yuan et al. This is an open access article distributed under the Creative Commons Attribution License, which permits unrestricted use, distribution, and reproduction in any medium, provided the original work is properly cited.

\begin{abstract}
Electrospinning is a simple and versatile technique for the fabrication of nonwoven fibrous materials for biomedical applications. In the present study, chitosan (CS) and polyethylene oxide (PEO) nanofibrous scaffolds were successfully prepared using three different CS/PEO mass ratios and then evaluated for their physical, chemical, and biological characteristics. Scaffold morphologies were observed by scanning electron microscopy, which showed decreasing fiber diameters with increasing CS content. Higher CS concentrations also correlated with increased tensile strength and decreased elasticity of the scaffold. Degradation studies demonstrated that PEO was solubilized from the scaffold within the first six hours, followed by CS. This profile was unaffected by changes in the CS/PEO ratio or the $\mathrm{pH}$ of the media. Only the $2: 1 \mathrm{CS} / \mathrm{PEO}$ scaffold demonstrated superior inhibition of both growth and attachment of Staphylococcus aureus. Finally, all scaffolds exhibited little impact on the proliferation of murine fibroblast monolayers. These data demonstrate that the $2: 1 \mathrm{CS} / \mathrm{PEO}$ scaffold is a promising candidate for wound dressing applications due to its excellent antibacterial characteristics and biocompatibility.
\end{abstract}

\section{Introduction}

Electrospinning is a technique used for the production of nanofiber scaffolds that exhibit high surface area, porosity, and characteristics that are biomimetic for the natural extracellular matrix. Furthermore, nanofibers produced by electrospinning are magnitudes smaller $(<1 \mu \mathrm{m})$ than those prepared by traditional methods of fiber fabrication $(>1 \mu \mathrm{m})$ [1]. Thus, electrospinning has emerged as one of the most widely used techniques to engineer the next generation of multifunctional nanofibrous scaffolds [2]. In electrospinning, a high voltage electric field (i.e., 10 to $30 \mathrm{kV}$ ) is used to generate a polymer jet that travels from the spinneret tip to a grounded collector. The production of nanofibers is dependent on both solution and electrospinning parameters, including the type of polymer solvent, solution viscosity, polymer concentration, conductivity, surface tension, intra/ intermolecular interactions, voltage, flow rate, flight distance, collector type, and spinneret diameter [3]. A number of different types of biopolymers and copolymers have been successfully electrospun, including poly(ethylene-co-vinyl alcohol) [4, 5], collagen [6], alginate [7], and collagenpolyethylene oxide [8].

Chitosan (CS), the N-deacetylated derivative of chitin obtained from crustacean shells and fungi by alkaline deacetylation [9], is a linear polymer composed of $\mathrm{N}$-acetyld-glucosamine units linked by $\beta$-D-(1-4) bonds. Chitosan has been used extensively in wound healing applications due to its intrinsic biocompatibility, antibacterial effects, and hemostatic properties [10, 11]. However, electrospinning pure CS solutions is hindered by its polycationic charge and inter/intrachain hydrogen bonding [12]. Recent reports have shown that polyethylene oxide (PEO) can greatly improve CS spinnability and that the resulting composite nanofibrous scaffolds are stable and noncytotoxic to cells [13-15]. 
Various blends of CS and PEO have been utilized as a delivery system for antibacterial agents in wound dressings $[13,16]$. However, there is limited information describing the effects of electrospinning on the inherent antibacterial properties of CS, or the impact of electrospun CS on bacterial attachment to the surface of scaffolds and wound dressings. Bacterial attachment and adhesion are integral steps in biofilm formation and culminate in the irreversible binding of the bacterium to the material surface. Biofilms allow bacteria to grow more efficiently and become resistant to antibiotic treatments, can disrupt the wound healing process, and have deleterious consequences on the wound environment. Therefore, it is imperative to understand if potential wound dressing materials impact bacterial attachment to the surface of the scaffold.

In this study, three different CS/PEO scaffold mass ratios were fabricated by electrospinning. The morphological, structural, and mechanical properties of the different mass ratios were analyzed by field emission scanning electron microscopy (SEM), Attenuated Total Reflectance Fourier Transform Infrared Spectroscopy (ATR-FTIR), and uniaxial tensile testing. The scaffolds were evaluated for their ability to inhibit both growth and attachment of Staphylococcus aureus (S. aureus). Finally, the biocompatibility of the scaffolds was evaluated.

\section{Materials and Methods}

2.1. Materials. Acetic acid (glacial, $\geq 99.85 \%$ ), chitosan (low molecular weight, 75-85\% deacetylation), polyethylene oxide (MW 300,000 g/mol), and Triton ${ }^{\mathrm{TM}} \mathrm{X}-100$ were purchased from Sigma-Aldrich (St. Louis, MO, USA). Dimethyl sulfoxide was purchased from EMD Millipore (Billerica, MA, USA). Mouse L929 fibroblasts, Staphylococcus aureus (25923), and cell culture reagents were purchased from American Type Culture Collection (Manassas, VA, USA).

\subsection{Electrospinning Conditions}

2.2.1. Preparation of CS/PEO Polymer Solutions. Chitosan/ PEO polymer blends with mass ratios of $2: 1,1: 1$, and $1: 2$ were made by dissolving CS and PEO in $45 \%$ (v/v) aqueous acetic acid to obtain a total polymer content of $5.5 \mathrm{wt} \%$. Triton X-100 (1\% v/v) and DMSO (10\% v/v) were added to the solutions and stirred overnight at $100 \mathrm{RPM}$ at room temperature. Solution viscosities were determined by DV-E viscometer (Brookfield, Middleboro, MA) at $25^{\circ} \mathrm{C}$ with an S18 spindle and torque ranges between 0.6 and 1.0 RPM. Conductivity was measured using a Malvern Zetasizer NanoZS (Worcestershire, UK).

2.2.2. Fabrication of CS/PEO Electrospun Nanofibers. Nanofibrous scaffolds were fabricated using the electrospinning scheme depicted in Figure 1. Polymer solutions were mechanically pumped into the electrospinning system at a flow rate of $0.7 \mathrm{~mL} / \mathrm{h}$ using an NE-1000 syringe pump (New Era Pump Systems, Farmingdale, NY) and a $30 \mathrm{~mL}$ disposable syringe connected to an 18-gauge spinneret. A DC voltage of $28 \mathrm{kV}$,

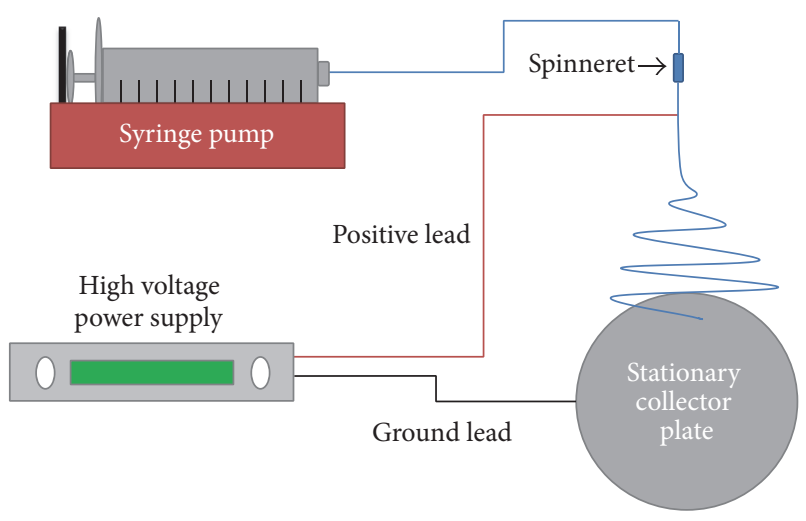

FIGURE 1: Schematic of the electrospinning system.

with a tip to collector distance of $22.5 \mathrm{~cm}$, was applied at the spinneret tip using a high voltage power supply (Spellman, Hauppauge, NY). For the bacterial, cell viability, and scaffold degradation assays, polymer scaffolds were deposited on $18 \mathrm{~mm}$ glass coverslips for seven hours at room temperature and a relative humidity of $16 \%$. For mechanical studies, scaffolds were deposited across an open $100 \mathrm{~mm}$ petri dish for 16 hours. Fabricated samples were stored in a desiccator at room temperature until analysis.

\subsection{Characterization of CS/PEO Electrospun Nanofibers}

2.3.1. Morphology. Electrospun nanofibrous scaffolds were sputter-coated with gold, and nanostructures were observed using field emission scanning electron microscopy (SEM) (Zeiss Sigma VP-40, Germany). Mean nanofiber diameters were calculated by measuring five random points per micrograph using Image ( $\mathrm{NIH}$, Bethesda, MD). Twenty micrographs were taken per scaffold, and three individually spun scaffolds were analyzed per formulation.

\subsubsection{Attenuated Total Reflectance Fourier Transform Infrared} Spectroscopy (ATR-FTIR). Infrared spectra were taken in transmission mode in the spectral range of 3500 to $500 \mathrm{~cm}^{-1}$ with a resolution of $4 \mathrm{~cm}^{-1}$, using a Spectrum 400 ATR-FTIR (PerkinElmer, Waltham, MA)

2.3.3. Mechanical Tensile Properties. Chitosan/PEO scaffolds were cut into $40 \mathrm{~mm} \times 25 \mathrm{~mm}$ rectangles, in accordance with the American Standards for Testing Methods D88212. Tensile testing was performed at a rate of $1 \mathrm{~mm} \mathrm{~min}^{-1}$ with a $250 \mathrm{~N}$ load cell and measured using Instron ${ }^{\circledR}$ E3000 Electropuls (Instron, Canton, MA). Young's moduli, ultimate stresses, and percent strain were calculated from the stressstrain curves.

2.4. In Vitro Scaffold Degradation. Chitosan/PEO nanofibrous scaffolds were placed in 12-well plates containing $300 \mu \mathrm{L}$ of $\mathrm{pH}$-adjusted $(6.5,7$, and 7.5) Eagle's Minimum Essential Medium (EMEM) and incubated at $37^{\circ} \mathrm{C}$ for 1 , 6,24 , or 72 hours. At the conclusion of each time point, 
assay supernatants were collected and analyzed by ATRFTIR. Additionally, the scaffolds were collected, washed with phosphate-buffered saline (PBS; pH 7.2), lyophilized, and weighed.

\subsection{Antibacterial Tests}

2.5.1. Bacterial Preparation and Maintenance. Staphylococcus aureus (S. aureus, ATCC 29523) was grown overnight in tryptic soy broth (Becton Dickinson, Franklin Lakes, NJ) at $37^{\circ} \mathrm{C}$ with continuous agitation. Initial seeding density was 3 $\times 10^{6}$ colony forming units $/ \mathrm{mL}$, which corresponded with a $600 \mathrm{~nm}$ optical density $\left(\mathrm{OD}_{600}\right)=0.1$.

2.5.2. Bacterial Planktonic Growth. Nanofibrous scaffolds $(n=9)$ were incubated statically with $S$. aureus at $37^{\circ} \mathrm{C}$. After 24 hours, bacterial samples were collected, serially diluted, plated on tryptic soy agar (TSA) plates (Becton Dickinson, Franklin Lakes, NJ), and incubated at $37^{\circ} \mathrm{C}$. Colony forming units $/ \mathrm{mL}$ were calculated by counting the number of colonies that developed after 24 hours. Changes in bacterial growth were calculated by normalizing results to the untreated control group.

2.5.3. Bacterial Attachment. Cultures of $S$. aureus were incubated statically with scaffolds $(n=9)$ in a 12 -well plate. After 24 hours, the scaffolds were washed twice with PBS (Invitrogen, Grand Island, NY) and sonicated for 30 seconds to remove any attached bacteria. Detached bacteria were collected, serially diluted, plated on TSA, and incubated at $37^{\circ} \mathrm{C}$. Colony forming units $/ \mathrm{mL}$ were calculated by counting the number of colonies that developed after 24 hours. The fold change in attachment was determined by comparing scaffoldtreated samples to a negative glass substrate control.

Bacterial attachment was visualized using Live/Dead ${ }^{\circledR}$ Baclight ${ }^{\mathrm{TM}}$ Bacteria Viability Kits (Invitrogen, Grand Island, NY) and imaged on a Nikon Eclipse C1 confocal laserscanning microscope (Nikon, Tokyo, Japan).

2.6. Biocompatibility. Indirect cytotoxicity of the scaffolds was evaluated based on an approach adapted from the ISO10993-5 standard test method [6, 17]. Mouse L929 fibroblasts were cultured at $37^{\circ} \mathrm{C}$ and $5 \% \mathrm{CO}_{2}$ in EMEM containing $10 \%$ horse serum. Media were refreshed every three days. Once the cells reached confluence, they were trypsinized and seeded into 24 -well plates $(25,000$ cells $/ \mathrm{mL})$. The following day, the media were replenished and nanofibrous scaffolds were introduced. Cell proliferation was monitored over 120 hours using a 2-(4-iodophenyl)-3-(4-nitrophenyl)5-(2,4-disulfophenyl)-2H-tetrazolium sodium (WST-1) cell proliferation assay.

Cellular supernatants were also examined for lactate dehydrogenase (LDH) at 24, 48, and 120 hours after scaffold exposure. $\mathrm{LDH}$ assay results were normalized to cells treated with $0.1 \%$ Triton X-100 as a positive control for cell death.

2.7. Statistics and Data Analysis. Statistical data were collected and presented as mean \pm standard deviation (SD).
TABLE 1: Viscosity of chitosan/PEO solutions generated with/without $10 \%$ DMSO and $1 \%$ Triton X-100.

\begin{tabular}{lcc}
\hline Mass ratio & Viscosity $(\mathrm{cP})$ & Conductivity $(\mathrm{mS} / \mathrm{cm})$ \\
\hline $2: 1$ without cosolvents & $4447 \pm 515$ & $2.15 \pm 0.04$ \\
$2: 1$ with DMSO only & $3557 \pm 142$ & $2.17 \pm 0.01$ \\
$2: 1$ with Triton X-100 & $2392 \pm 120^{*}$ & $2.52 \pm 0.03^{\#}$ \\
$2: 1$ with cosolvents & $3067 \pm 738^{*}$ & $2.19 \pm 0.19$ \\
$1: 1$ with cosolvents & $2044 \pm 413^{*}$ & $1.67 \pm 0.07^{\#}$ \\
$1: 2$ with cosolvents & $1871 \pm 254^{*}$ & $1.24 \pm 0.05^{\#}$ \\
\hline
\end{tabular}

${ }^{*} p<0.05$, viscosity comparisons made to $2: 1 \mathrm{CS} / \mathrm{PEO}$ without cosolvents. ${ }^{\#} p<0.05$, conductivity comparisons made to $2: 1 \mathrm{CS} / \mathrm{PEO}$ without cosolvents.

Data were analyzed and compared using analysis of variance (ANOVA) on Graphpad Prism (San Diego, CA, USA). Multiple comparisons were made among groups using Tukey's multiple comparison tests. Statistical significance was determined at $p<0.05(95 \% \mathrm{CI})$.

\section{Results and Discussion}

3.1. Electrospinning and Morphology of CS/PEO Nanofibers. Scaffold microstructures were observed by SEM and revealed that the CS/PEO mass ratios $2: 1,1: 1$, and $1: 2$ generated fiber diameters of $244 \pm 70 \mathrm{~nm}, 289 \pm 47 \mathrm{~nm}$, and $360 \pm$ $62 \mathrm{~nm}$, respectively (Figure 2). Although the total polymer concentration remained constant for all three CS/PEO ratios, changes in fiber diameters directly correlated with the CS concentration. Higher CS concentrations may increase the charge density of the polymer solution, resulting in more bending of ejected jet [18]. This, in turn, would produce a thinner fiber with increased solvent evaporation. Additionally, lower PEO concentrations are known to increase the conductivity and viscosity of the polymer solutions, which could also contribute to formation of thinner nanofibers (Table 1) $[15,19,20]$.

The viscosity of a polymer solution is determined by the intermolecular interactions between polymer chains. Strong hydrogen bonding between $\mathrm{NH}_{2}$ and $\mathrm{OH}$ groups of CS chains results in high viscosity solutions that are unsuitable for electrospinning [21, 22]. In addition, the polycationic properties of pure CS make it difficult to electrospin. Thus, the addition of copolymers and solvents is necessary to impart more favorable electrospinning properties to the polymer solution [23]. Polyethylene oxide can interact with CS molecules by disrupting its self-association, which improves CS solubility and lowers the overall viscosity of the blended polymer solution [23]. As such, PEO was utilized as a copolymer for CS scaffold fabrication. However, even with the addition of PEO, it was found that solutions with total polymer concentrations greater than $5.5 \%$ resulted in high viscosity solutions that could not be electrospun.

Use of polar or nonpolar cosolvents is another commonly used technique to improve electrospinnability [21]. Without the addition of Triton X-100 and DMSO as a surfactant and cosolvent, respectively, CS/PEO solutions with ratio greater than 1:1 did not produce smooth nanofibers when 


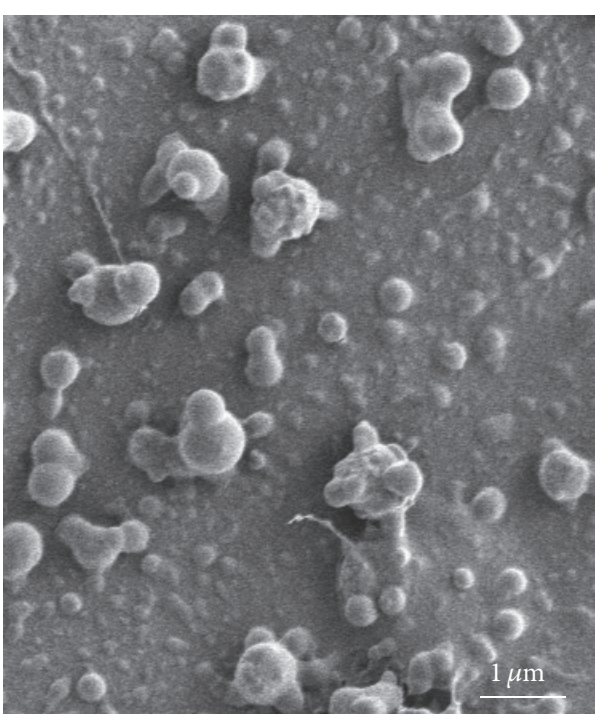

(a)

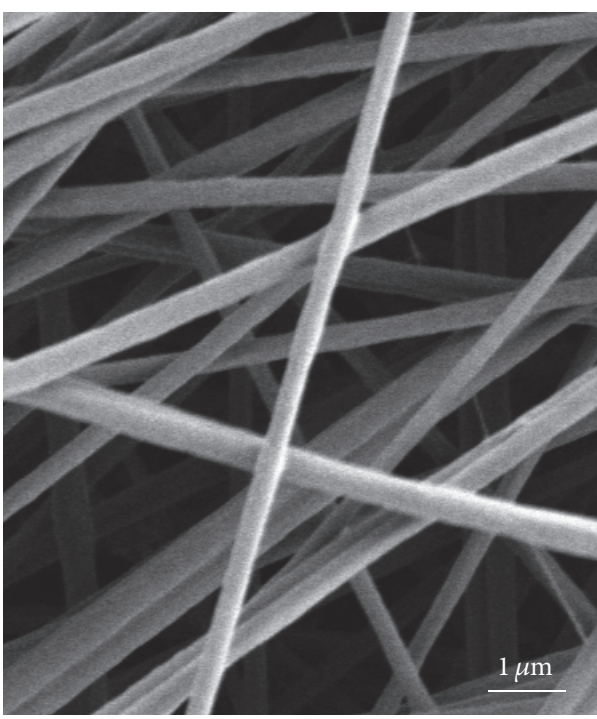

(c)

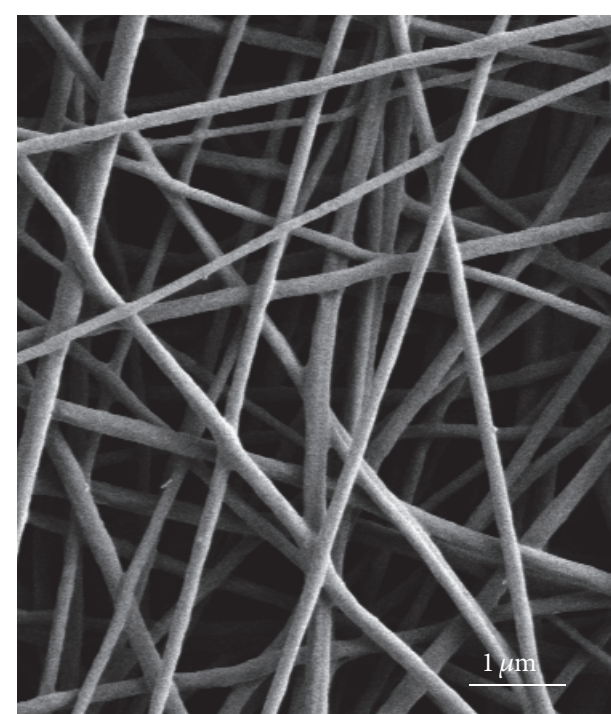

(b)

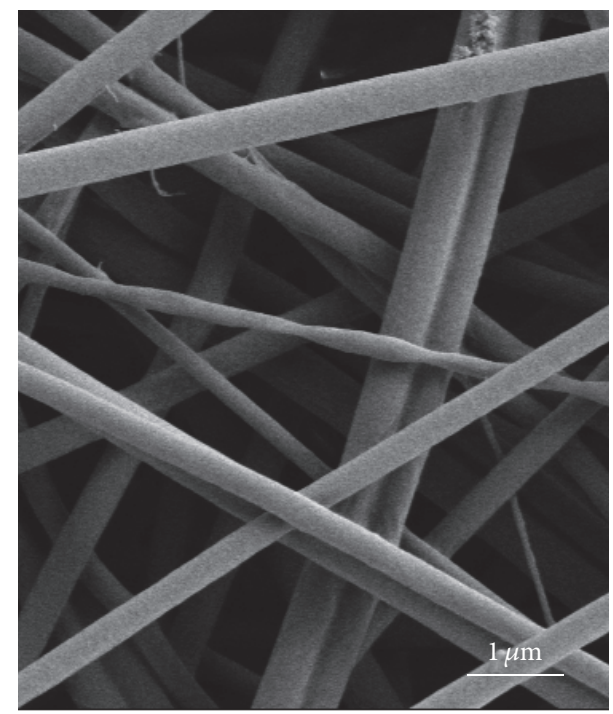

(d)

FIGURE 2: Representative SEM micrographs of electrospun scaffolds. (a) 2:1 CS/PEO without Triton X-100/DMSO. (b) $2: 1$ CS/PEO scaffold with Triton X-100/DMSO and mean diameter $244 \pm 70 \mathrm{~nm}$. (c) $1: 1 \mathrm{CS} / \mathrm{PEO}$ scaffold with Triton X-100/DMSO and mean diameter $289 \pm 47 \mathrm{~nm}$. (d) $1: 2 \mathrm{CS} / \mathrm{PEO}$ scaffold with Triton X-100/DMSO and mean diameter $360 \pm 62 \mathrm{~nm}$.

electrospun (Figure 2(a)). Surfactants such as Triton X100 can modulate intramolecular interactions and lower the viscosity of various polymer solutions, including $\mathrm{CS} / \mathrm{PEO}$ solutions (Table 1) [23]. Although DMSO did not impact the viscosity or conductivity of the CS/PEO formulations, DMSO has been shown to relax chain entanglements and improve solution volatility [22]. As such, both Triton X100 and DMSO are important components in CS/PEO and comparable fabrications [21].

3.2. Composition of CS/PEO Nanofibers. Attenuated Total Reflectance Fourier Transform Infrared Spectroscopy was utilized to confirm scaffold compositions and investigate potential interactions between CS and PEO (Figure 3). Similar to pure CS solutions, the ATR-FTIR spectra for the CS/PEO blended scaffold showed a characteristic broad band around $3262 \mathrm{~cm}^{-1}$, which is attributed to the stretching vibrations of $-\mathrm{NH}_{2}$ groups and is typically broad for CS with high degrees of deacetylation [24]. The peaks at $1544 \mathrm{~cm}^{-1}$ and $1407 \mathrm{~cm}^{-1}$ were due to the carbonyl stretching of the amide bands and the $\mathrm{N}-\mathrm{H}$ bending of the CS amino groups, respectively. Similar to pure PEO solutions, the electrospun $\mathrm{CS} / \mathrm{PEO}$ scaffold also showed two distinct sharp bands at $1100 \mathrm{~cm}^{-1}$ and $2882 \mathrm{~cm}^{-1}$, which characteristically represent ether groups (C-O-C) and the associated $\mathrm{Sp}^{3} \mathrm{C}-\mathrm{H}$ stretches, respectively.

While these data clearly demonstrate that the CS/PEO scaffolds shared characteristic peaks with both CS and PEO polymers, hydrogen bonds were also expected to form among the CS hydroxyl groups $(-\mathrm{OH})$, the CS carbonyl groups 


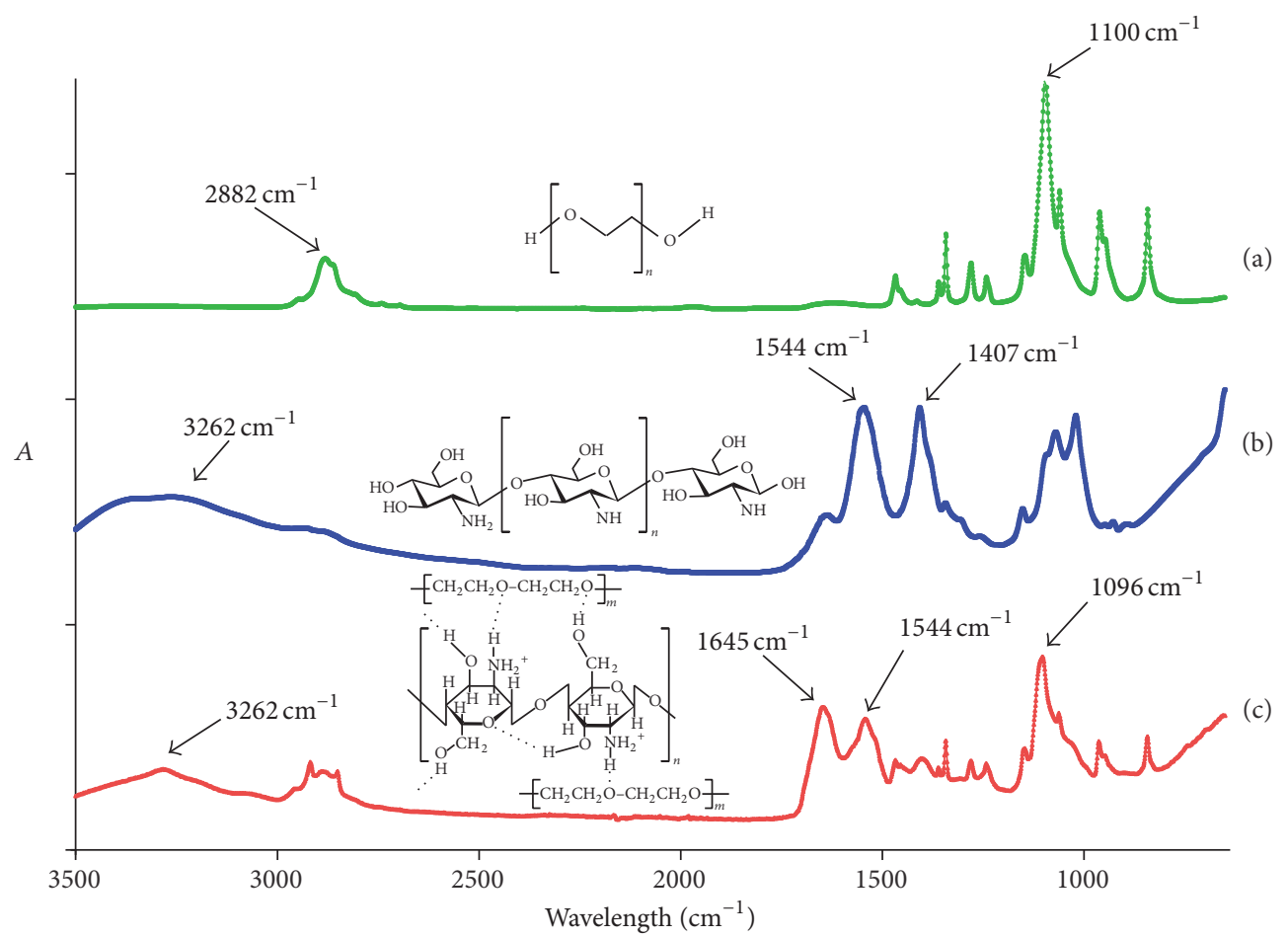

FIGURE 3: Representative ATR-FTIR spectra of (a) PEO in solution, (b) CS in solution, and (c) a representative electrospun 1:1 CS/PEO scaffold. Insets: structures of (a) PEO, (b) CS, and (c) CS/PEO.

$(\mathrm{C}=\mathrm{O}-\mathrm{NHR})$, the $\mathrm{CS}$ amine groups $\left(-\mathrm{NH}_{2}\right)$, and the PEO ether groups (C-O-C) $[19,21]$. This was confirmed by the spectra of CS/PEO scaffolds, which displayed unique ATRFTIR peaks. Notably, the appearance of a peak at $1645 \mathrm{~cm}^{-1}$ is associated with the presence of a carbonyl stretch that was absent from the pure CS spectra. The characteristic stretching of the PEO ether (C-O-C) group also shifted to a lower wavelength (from $1100 \mathrm{~cm}^{-1}$ to $1096 \mathrm{~cm}^{-1}$ ). Together, these changes in the ATF-FTIR spectra are indicative of hydrogen bonding between polyether oxygen and amino hydrogen groups in PEO and CS.

3.3. Mechanical Tensile Properties. Tensile strength describes the amount of stress that a material can bear before suffering permanent deformations and can be used to quantify the overall intermolecular fiber connectivity strength of the nanofibrous scaffolds. Conversely, Young's Modulus measures the flexibility of the material and can be used to describe a material's resistance to being deformed elastically.

Natural CS is rigid and brittle, which can be attributed to strong intra- and intermolecular hydrogen bonds in the pyranose backbone of CS. Conversely, PEO chains are flexible due to their linear structure and lack of bulky side groups. Therefore, decreasing the CS content and increasing the PEO ratio within the CS/PEO scaffolds were expected to decrease the strength of the scaffold but increase the overall flexibility of the blended scaffold.

Uniaxial tensile testing was performed on CS/PEO blended nanofibrous scaffolds with a thickness of $125 \pm$ $3.4 \mu \mathrm{m}$. The respective tensile strength of the scaffold
TABLE 2: Tensile properties of electrospun CS/PEO scaffolds with increasing mass ratio of chitosan $(n=9)$.

\begin{tabular}{lccc}
\hline Mass ratio $\begin{array}{c}\text { Young's Modulus } \\
(\mathrm{MPa})\end{array}$ & $\begin{array}{c}\text { Tensile strength } \\
(\mathrm{MPa})\end{array}$ & $\begin{array}{c}\text { Strain at break } \\
(\%)\end{array}$ \\
\hline $2: 1$ & $24.6 \pm 6.5$ & $2.8 \pm 1.0$ & $14.7 \pm 2.5$ \\
$1: 1$ & $14.0 \pm 3.5^{*}$ & $1.1 \pm 0.5^{\#}$ & $10.7 \pm 4.6$ \\
$1: 2$ & $9.1 \pm 4.2^{*}$ & $0.9 \pm 0.4^{\#}$ & $11.7 \pm 3.6$ \\
\hline
\end{tabular}

${ }^{*} p<0.05$, Young's Modulus comparisons made to $2: 1$ mass ratio scaffolds. $\# p<0.05$, tensile strength comparisons made to $2: 1$ mass ratio scaffolds.

increased from $0.9 \pm 0.4$ to $1.1 \pm 0.5$ to $2.8 \pm 1.0 \mathrm{MPa}$, which correlated with increasing CS concentrations (Table 2, Figure 4). By doubling the amount of chitosan from the $1: 1$ to $2: 1$, scaffolds significantly increased both Young's Modulus and tensile strength. These data suggest that higher CS concentrations were associated with an increase in overall strength and a decrease in scaffold elasticity. No significant differences were observed in the percent strain at break of the scaffolds. From these results, it can be concluded that higher CS content increased scaffold strength and decreased scaffold elasticity under uniaxial tension.

3.4. In Vitro Scaffold Degradation. It is well established that the wound environment can vary significantly in $\mathrm{pH}$, depending on the nature of the wound. For example, studies have shown that the environment tends to be more basic in chronic wounds ( $\mathrm{pH} 7.3-8.9$ ) [25], while normal healing wounds have acidic to neural $\mathrm{pH}$ levels throughout the healing process 


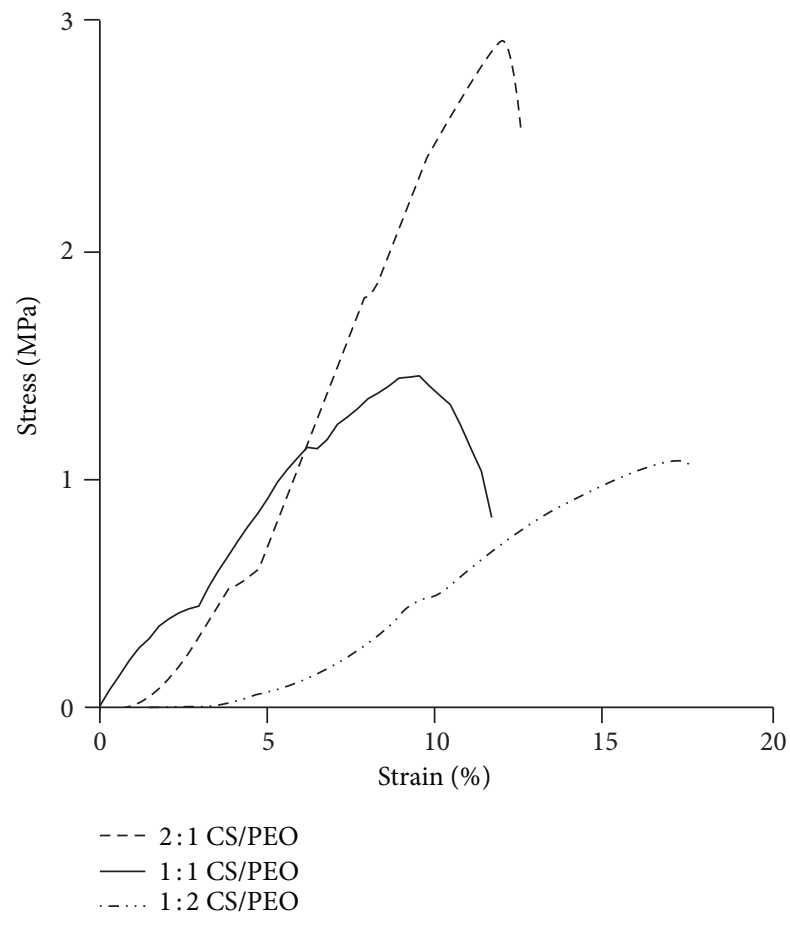

FIGURE 4: Representative stress-strain curves of different mass ratios of CS/PEO scaffolds. Data is representative of five independently produced scaffolds.

(pH 5.8-7.5) [26]. Therefore, it is important to understand how varying $\mathrm{pH}$ levels might impact biodegradable wound dressings. However, there is little evidence of how varying $\mathrm{pH}$ conditions affect $\mathrm{CS}$ and $\mathrm{PEO}$ release from electrospun CS/PEO scaffolds.

Nanofiber degradation was evaluated by incubation of scaffold samples in $\mathrm{pH}$-adjusted media ( $\mathrm{pH} 6.5,7.0$, or 7.5) at $37^{\circ} \mathrm{C}$ for 72 hours. This was followed by SEM, dry weight analysis, and identification of soluble products. It is known that CS solubility is highly dependent on the $\mathrm{pH}$ of the solvent while PEO is readily soluble in most aqueous solutions. Thus, it was expected that PEO and CS would solubilize faster in more acidic environments, resulting in a larger weight loss. However, $\mathrm{pH}$ changes between 6.5 and $7.5 \mathrm{did}$ not significantly impact the weight loss of the scaffold over 72 hours.

The morphology of the degraded fibers was evaluated by SEM (Figure 5). After being incubated for 72 hours, the $2: 1 \mathrm{CS} / \mathrm{PEO}$ mass ratio scaffold maintained its nanofiber structure. However, there was an observable loss of nanofiber morphology for the $1: 1$ and $1: 2$ CS/PEO mass ratio scaffolds. These observations demonstrate that scaffolds with higher PEO concentrations exhibited higher solubility in aqueous solutions, leading to the loss of structural morphology.

To examine the amount of polymer loss from the scaffold at each of the prescribed time points, scaffolds were dried and weighed before and after each time point. The weight was described as a percent of the remaining scaffold at each time point (Figure 6(a)). It is likely that the solubility of PEO contributed to the majority of the scaffold weight loss

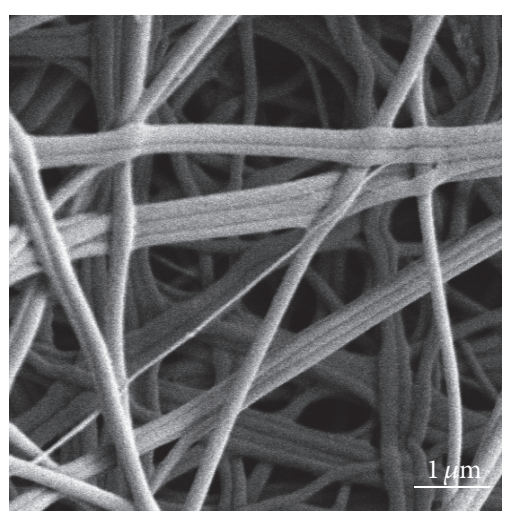

(a)

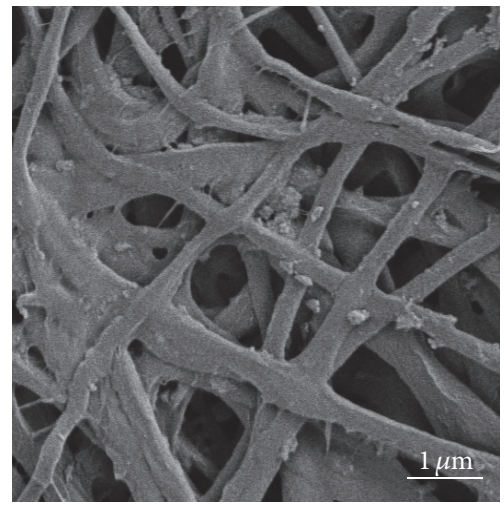

(b)

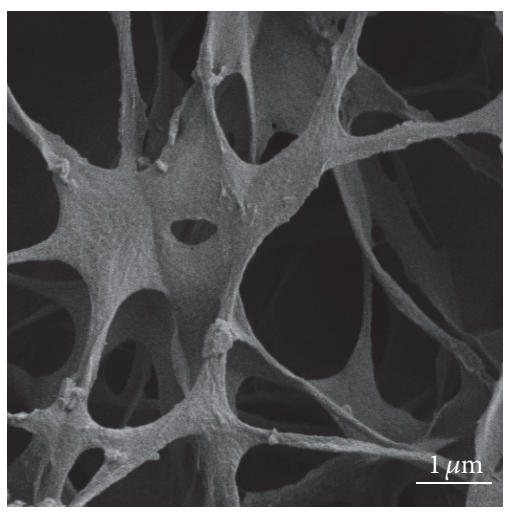

(c)

FIGURE 5: Representative SEM micrographs of (a) $2: 1$, (b) $1: 1$, and (c) $1: 2 \mathrm{CS} / \mathrm{PEO}$ scaffolds after 72 hours of incubation in $\mathrm{pH} 7.0$ media.

in the first hour. Due to the solubility of PEO, it would be expected that the decrease in weight would be proportional to the increase in PEO. However, the three CS/PEO mass ratios displayed similar weight loss trends, suggesting that the CS-PEO interaction alters PEO and/or CS solubility (data not shown). Additionally, $\mathrm{pH}$ did not significantly impact the weight loss of the scaffold over 72 hours (data not shown).

To further confirm the release of PEO and the potential solubilization of CS from the scaffold, supernatants were analyzed by ATR-FTIR. The characteristic PEO ether (C-OC) stretch for PEO at $1096 \mathrm{~cm}^{-1}$ could be observed within the 


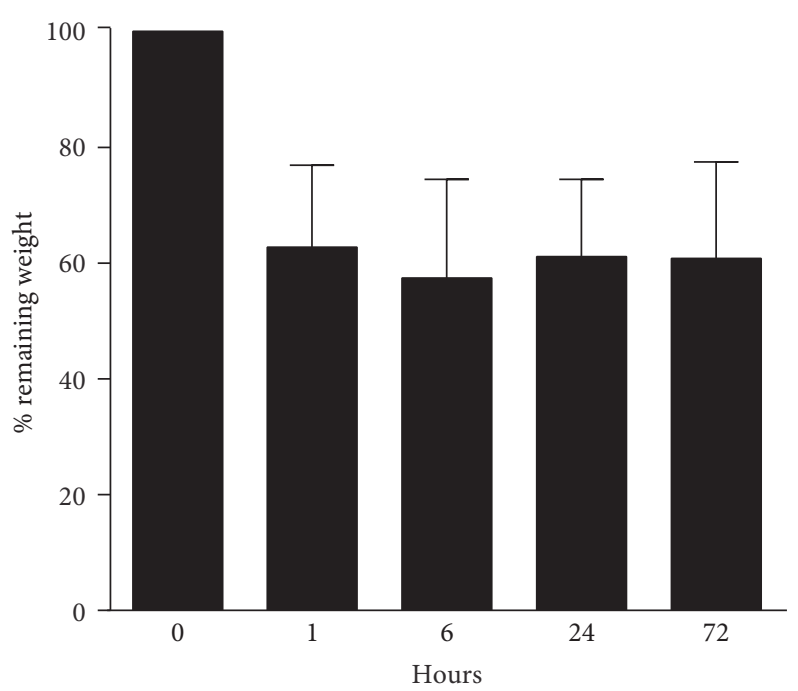

(a)

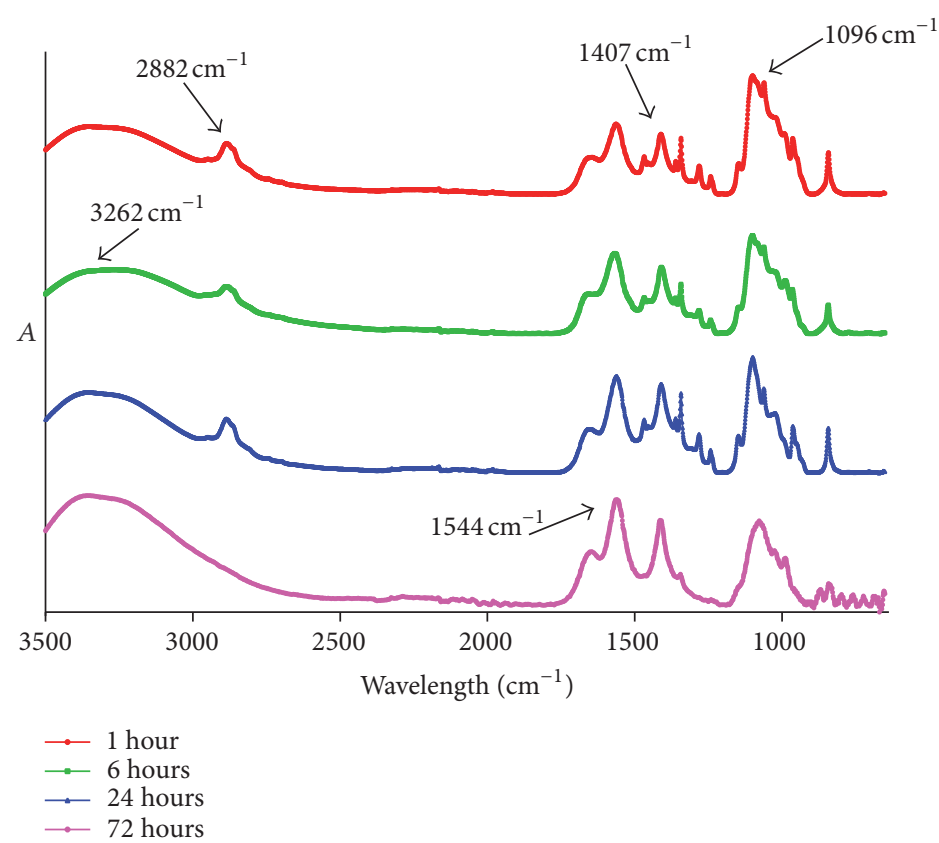

(b)

FIGURE 6: (a) Dry weight loss and corresponding (b) ATR-FTIR spectra of supernatants from 1:1 CS/PEO scaffolds placed in EMEM (pH 7) at $37^{\circ} \mathrm{C}$. Error bars represent mean \pm SD. $n=9$.

first hour and remained relatively unchanged throughout the 72 hours for all scaffolds evaluated (Figure 6(b)). However, the associated PEO Sp ${ }^{3} \mathrm{C}-\mathrm{H}$ stretch at $2882 \mathrm{~cm}^{-1}$ was undetectable at 72 hours. In addition, characteristic CS carbonyl $\left(1544 \mathrm{~cm}^{-1}\right)$ and $-\mathrm{NH}_{2}$ group $\left(1407 \mathrm{~cm}^{-1}\right)$ stretches were also detected in the first hour and increased by 72 hours relative to the $1096 \mathrm{~cm}^{-1}$ peak, which remained relatively constant. Together, these results suggest that the observed weight loss might be attributed to PEO solubilization and that the hydrogen bond between PEO and CS may facilitate CS release.

3.5. Antibacterial Properties of Electrospun CS/PEO. Although the antimicrobial effects of CS-based nanofiber scaffolds on bacteria growth have been reported, there are few reports on how CS-based nanofiber scaffolds impact bacterial attachment [16]. Bacterial attachment/adhesion is an integral step in biofilm formation and culminates in the irreversible binding of the bacterium to the material surface. Biofilm formation allows the bacteria to grow more efficiently and become more resistant to antibiotic treatments. For wound dressing applications, it is important to inhibit this initial attachment step to prevent biofilm formation.

To measure planktonic growth and bacterial attachment, S. aureus was exposed to the CS/PEO scaffolds for 24 hours. The $2: 1 \mathrm{CS} / \mathrm{PEO}$ nanofibrous scaffolds reduced planktonic bacterial loads by $90 \%$ compared to an untreated control. Conversely, 1:1 and 1:2 CS/PEO scaffolds had minimal effect on $S$. aureus growth (Figure 7(a)). 


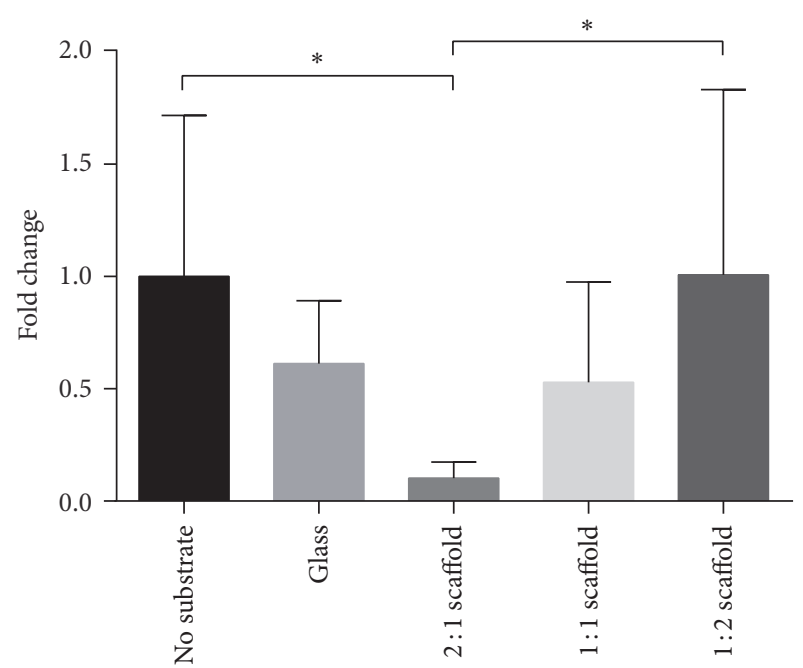

(a)

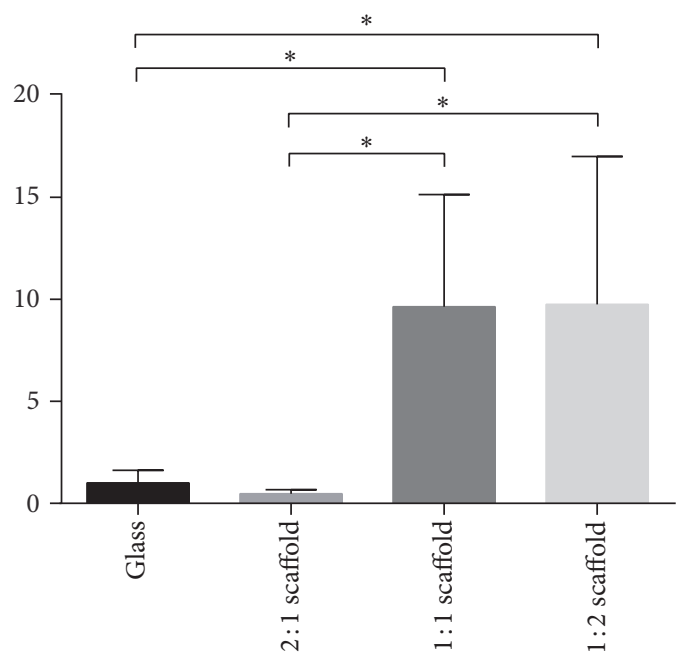

(b)

FIGURE 7: Fold changes in bacterial (a) growth and (b) attachment after being exposed to different mass ratios of electrospun CS/PEO nanofibrous scaffolds for 24 hours. Error bars represent mean \pm SD. $n=9,{ }^{*} p<0.05$.

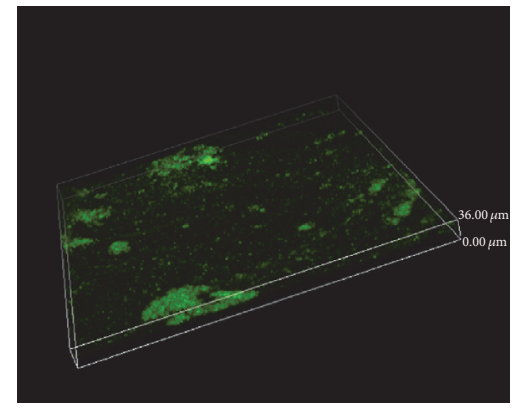

(a)

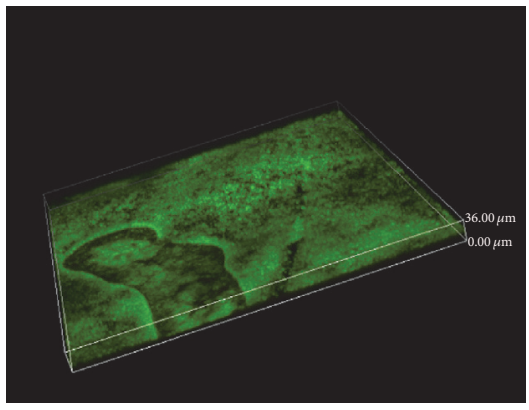

(b)

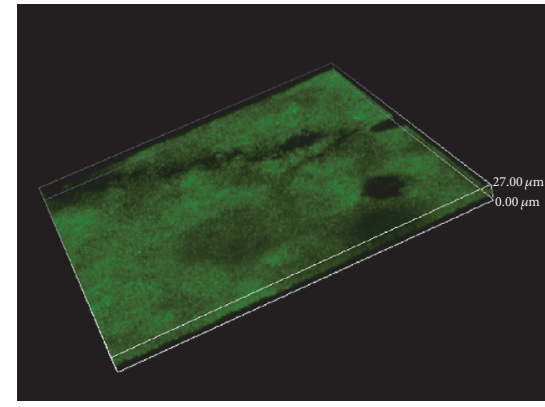

(c)

FIGURE 8: Confocal micrographs of $S$. aureus attached to $2: 1$ (a), 1:1 (b), and 1:2 (c) CS/PEO nanofibrous scaffolds. Image representative of six replicates.

Scaffolds were then collected to assess bacterial attachment. The $2: 1$ mass ratio CS/PEO nanofibrous scaffolds had significantly fewer bacterial colonies attached to the surface compared to $1: 1$ and 1:2 mass ratio scaffolds (Figure 7(b)). Visualization of bacterial attachment to the three electrospun mass ratios of CS/PEO by confocal microscopy confirmed that only the $2: 1 \mathrm{CS} / \mathrm{PEO}$ scaffold notably reduced bacterial attachment (Figure 8 ).

There are various mechanisms by which the $2: 1 \mathrm{CS} / \mathrm{PEO}$ scaffold may induce these antibacterial properties. Chitosan is known to have antimicrobial action due to amine groups on the $\mathrm{C} 2$ atoms. Therefore, free solubilized CS could interact with the negatively charged groups on the surface of the bacteria and alter bacterial membrane permeability [9]. Additionally, CS has been shown to interact with bacterial DNA to impede bacterial replication $[9,11]$. There is also evidence that CS can inhibit bacterial attachment by blocking putative cell attachment sites [27] or by indirectly blocking bacterial attachment proteins [9]. Finally, it is possible that the reduction in bacterial attachment might be a direct result of solubilized CS that is released from the scaffold. Thus, a higher concentration of released CS was expected to enhance the antibacterial capacity of the scaffold.

3.6. Biocompatibility. To examine the CS/PEO scaffolds for a putative use as a wound dressing, scaffolds were evaluated for biocompatibility with L929 murine fibroblast cells for up to 120 hours. No discernable differences in cellular proliferation were detected between cells exposed to the scaffolds or the negative control (Figure 9). To examine potential toxic effects of the CS/PEO scaffolds, cell supernatants were also tested for the presence of lactate dehydrogenase (LDH), an intracellular enzyme that is released when cellular membranes are compromised. Only the Triton X-100 treatment significantly impacted L929 viability compared to the substrate control. This demonstrates that although CS/PEO scaffolds were capable of affecting the growth and/or attachment of bacteria cells, neither the scaffolds nor the scaffold release products significantly impacted fibroblast growth or viability. 


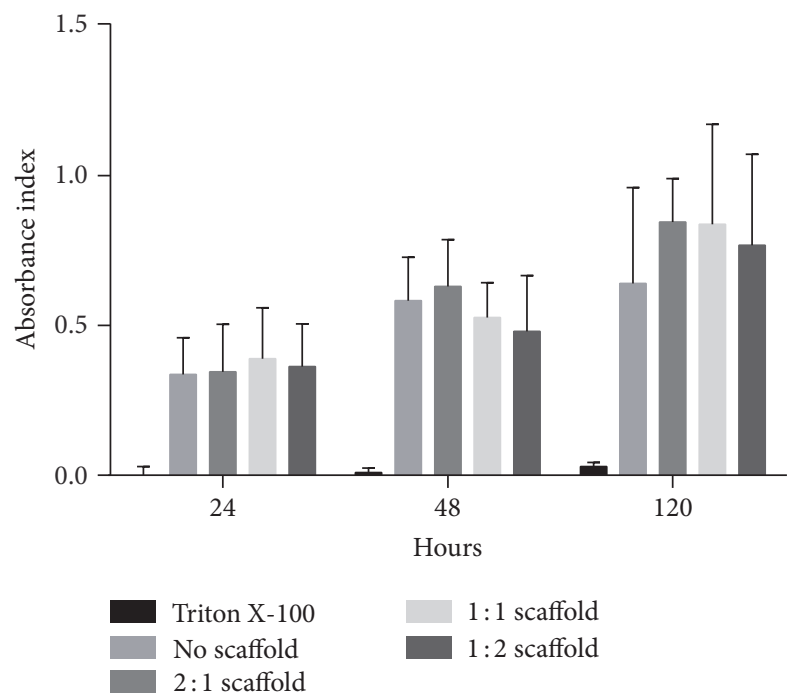

(a)

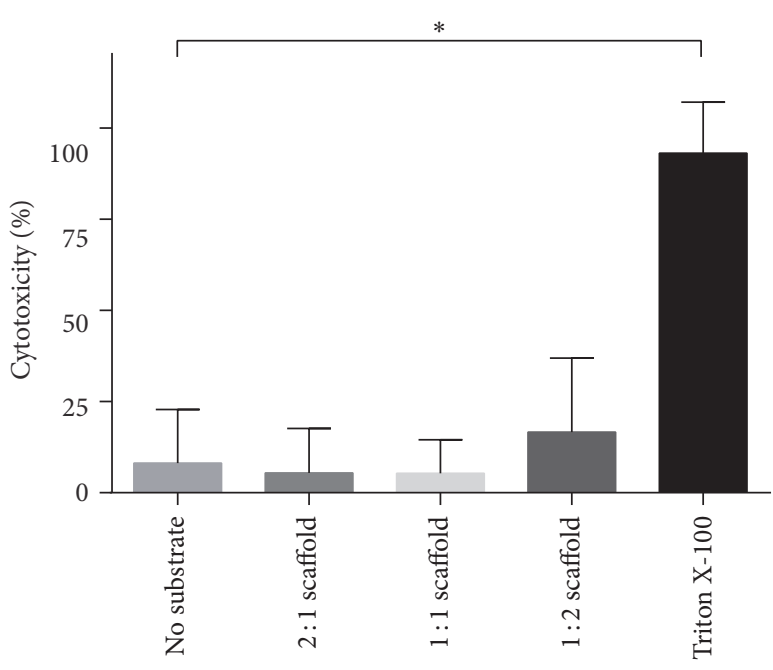

(b)

FIGURE 9: Comparison of L929 fibroblast (a) proliferation and (b) cytotoxicity over 72 hours in the presence of CS/PEO nanofibrous scaffolds. Error bars represent mean \pm SD. $n=8,{ }^{*} p<0.05$.

\section{Conclusions}

Electrospun CS/PEO nanofibrous scaffolds were characterized by examining scaffold (1) morphology, (2) physical and mechanical properties, (3) degradation profiles, (4) antibacterial properties, and (5) toxicity to mammalian cells. Changes in CS content impacted fiber diameters and scaffold tensile strength and flexibility. Degradation studies suggest that the early mass loss was associated with the release of both CS and PEO from the scaffolds. Antibacterial studies demonstrated that the 2:1 mass ratio CS/PEO scaffold imparted a stronger inhibition of $S$. aureus growth and attachment than the other CS/PEO scaffolds. Finally, it was determined that neither the scaffold nor the release of scaffold degradation products exhibited significant cytotoxic effects against murine fibroblast cells. The 2:1 scaffolds demonstrated excellent antibacterial properties in respect to inhibiting $S$. aureus attachment to the surface of the scaffold. This property could play an important role in wound dressing applications, where infection is often introduced through attachment and infiltration of bacteria during dressing changes. Overall, these results demonstrate that CS/PEO scaffolds with at least a 2:1 mass ratio are well suited to serve as a foundation for the development of a next generation antimicrobial wound dressing.

\section{Disclosure}

The views expressed in this article are those of the authors and do not necessarily reflect the official policy or position of the Department of the Navy, Department of Defense, or the US Government. The authors are military service members or government or contract employees of the US Government. This work was prepared as part of their official duties. Title 17 U.S.C. $\$ 105$ provides that "Copyright protection under this title is not available for any work of the United States
Government.” Title 17 U.S.C. $\$ 101$ defines a US Government work as a work prepared by a military service member or employee of the US Government as part of that person's official duties.

\section{Competing Interests}

The authors declare that they have no competing interests.

\section{Authors' Contributions}

Tony T. Yuan, Phillip M. Jenkins, and Ann Marie DiGeorge Foushee designed the experiments. Tony T. Yuan and Phillip M. Jenkins prepared the electrospun scaffolds and performed degradation experiments. Angela R. Jockheck-Clark and Jonathan M. Stahl carried out analysis of the degradation data. Ann Marie DiGeorge Foushee performed and analyzed cell toxicity experiments. Tony T. Yuan performed and analyzed antibacterial experiments. Tony T. Yuan, Phillip M. Jenkins, Ann Marie DiGeorge Foushee, Angela R. JockheckClark, and Jonathan M. Stahl prepared the manuscript.

\section{Acknowledgments}

The authors would like to thank Dr. Tao You of the US Army Institute of Surgical Research for his valuable contributions on the SEM studies. This work was funded by the Naval Medical Research Center's Advanced Medical Development Program supported by work unit number G1406.

\section{References}

[1] J. M. Deitzel, J. D. Kleinmeyer, J. K. Hirvonen, and N. C. Beck Tan, "Controlled deposition of electrospun poly(ethylene oxide) fibers," Polymer, vol. 42, no. 19, pp. 8163-8170, 2001. 
[2] Z. Xie, C. B. Paras, H. Weng et al., "Dual growth factor releasing multi-functional nanofibers for wound healing," Acta Biomaterialia, vol. 9, no. 12, pp. 9351-9359, 2013.

[3] D. I. Braghirolli, D. Steffens, and P. Pranke, "Electrospinning for regenerative medicine: a review of the main topics," Drug Discovery Today, vol. 19, no. 6, pp. 743-753, 2014.

[4] E.-R. Kenawy, J. M. Layman, J. R. Watkins et al., "Electrospinning of poly(ethylene-co-vinyl alcohol) fibers," Biomaterials, vol. 24, no. 6, pp. 907-913, 2003.

[5] B. Wang, C. Xu, F. Xu, and T. Lu, "Electrospinning of poly(ethylene-co-vinyl alcohol) nanofibres encapsulated with Ag nanoparticles for skin wound healing," Journal of Nanomaterials, vol. 2011, Article ID 201834, 7 pages, 2011.

[6] J.-P. Chen, G.-Y. Chang, and J.-K. Chen, "Electrospun collagen/chitosan nanofibrous membrane as wound dressing," Colloids and Surfaces A: Physicochemical and Engineering Aspects, vol. 313-314, pp. 183-188, 2008.

[7] S. I. Jeong, M. D. Krebs, C. A. Bonino, S. A. Khan, and E. Alsberg, "Electrospun alginate nanofibers with controlled cell adhesion for tissue engineering," Macromolecular Bioscience, vol. 10, no. 8, pp. 934-943, 2010.

[8] L. Huang, K. Nagapudi, P. R. Apkarian, and E. L. Chaikof, "Engineered collagen-PEO nanofibers and fabrics," Journal of Biomaterials Science, Polymer Edition, vol. 12, no. 9, pp. 979-993, 2001.

[9] E. I. Rabea, M. E.-T. Badawy, C. V. Stevens, G. Smagghe, and W. Steurbaut, "Chitosan as antimicrobial agent: applications and mode of action," Biomacromolecules, vol. 4, no. 6, pp. 1457-1465, 2003.

[10] X. Huang, Y. Sun, J. Nie et al., "Using absorbable chitosan hemostatic sponges as a promising surgical dressing," International Journal of Biological Macromolecules, vol. 75, pp. 322-329, 2015.

[11] M. Kong, X. G. Chen, K. Xing, and H. J. Park, “Antimicrobial properties of chitosan and mode of action: a state of the art review," International Journal of Food Microbiology, vol. 144, no. 1, pp. 51-63, 2010.

[12] H. Homayoni, S. A. H. Ravandi, and M. Valizadeh, "Electrospinning of chitosan nanofibers: processing optimization," Carbohydrate Polymers, vol. 77, no. 3, pp. 656-661, 2009.

[13] J. An, H. Zhang, J. Zhang, Y. Zhao, and X. Yuan, "Preparation and antibacterial activity of electrospun chitosan/ poly(ethylene oxide) membranes containing silver nanoparticles," Colloid and Polymer Science, vol. 287, no. 12, pp. 1425-1434, 2009.

[14] B. Duan, C. Dong, X. Yuan, and K. Yao, "Electrospinning of chitosan solutions in acetic acid with poly (ethylene oxide)," Journal of Biomaterials Science, Polymer Edition, vol. 15, no. 6, pp. 797-811, 2004.

[15] A. Subramanian, D. Vu, G. F. Larsen, and H.-Y. Lin, "Preparation and evaluation of the electrospun chitosan/PEO fibers for potential applications in cartilage tissue engineering," Journal of Biomaterials Science, Polymer Edition, vol. 16, no. 7, pp. 861-873, 2005.

[16] R. Erdem and M. Akalın, "Characterization and evaluation of antimicrobial properties of electrospun chitosan/polyethylene oxide based nanofibrous scaffolds (with/without nanosilver)," Journal of Industrial Textiles, vol. 44, no. 4, pp. 553-571, 2015.

[17] N. Charernsriwilaiwat, P. Opanasopit, T. Rojanarata, and T. Ngawhirunpat, "Lysozyme-loaded, electrospun chitosan-based nanofiber mats for wound healing," International Journal of Pharmaceutics, vol. 427, no. 2, pp. 379-384, 2012.
[18] M. M. Hohman, M. Shin, G. Rutledge, and M. P. Brenner, "Electrospinning and electrically forced jets. II. Applications," Physics of Fluids, vol. 13, no. 8, pp. 2221-2236, 2001.

[19] M. Pakravan, M.-C. Heuzey, and A. Ajji, "A fundamental study of chitosan/PEO electrospinning," Polymer, vol. 52, no. 21, pp. 4813-4824, 2011.

[20] M. Spasova, N. Manolova, D. Paneva, and I. Rashkov, "Preparation of chitosan-containing nanofibres by electrospinning of chitosan/poly(ethylene oxide) blend solutions," E-Polymers, vol. 4, no. 1, pp. 624-635, 2004.

[21] S. Zivanovic, J. Li, P. M. Davidson, and K. Kit, "Physical, mechanical, and antibacterial properties of chitosan/PEO blend films," Biomacromolecules, vol. 8, no. 5, pp. 1505-1510, 2007.

[22] N. Bhattarai, D. Edmondson, O. Veiseh, F. A. Matsen, and M. Zhang, "Electrospun chitosan-based nanofibers and their cellular compatibility," Biomaterials, vol. 26, no. 31, pp. 61766184, 2005.

[23] C. Kriegel, K. M. Kit, D. J. McClements, and J. Weiss, “Electrospinning of chitosan-poly(ethylene oxide) blend nanofibers in the presence of micellar surfactant solutions," Polymer, vol. 50, no. 1, pp. 189-200, 2009.

[24] Z. Zakaria, Z. Izzah, M. Jawaid, and A. Hassan, "Effect of degree of deacetylation of chitosan on thermal stability and compatibility of chitosan-polyamide blend," BioResources, vol. 7, no. 4, pp. 5568-5580, 2012.

[25] K. Rumbaugh, C. Watters, and T. Yuan, "Beneficial and deleterious bacterial-host interactions in chronic wound pathophysiology," Chronic Wound Care Management and Research, vol. 2, pp. 53-62, 2015.

[26] L. A. Schneider, A. Korber, S. Grabbe, and J. Dissemond, "Influence of $\mathrm{pH}$ on wound-healing: a new perspective for wound-therapy?" Archives of Dermatological Research, vol. 298, no. 9, pp. 413-420, 2007.

[27] J. Palmer, S. Flint, and J. Brooks, "Bacterial cell attachment, the beginning of a biofilm," Journal of Industrial Microbiology \& Biotechnology, vol. 34, no. 9, pp. 577-588, 2007. 

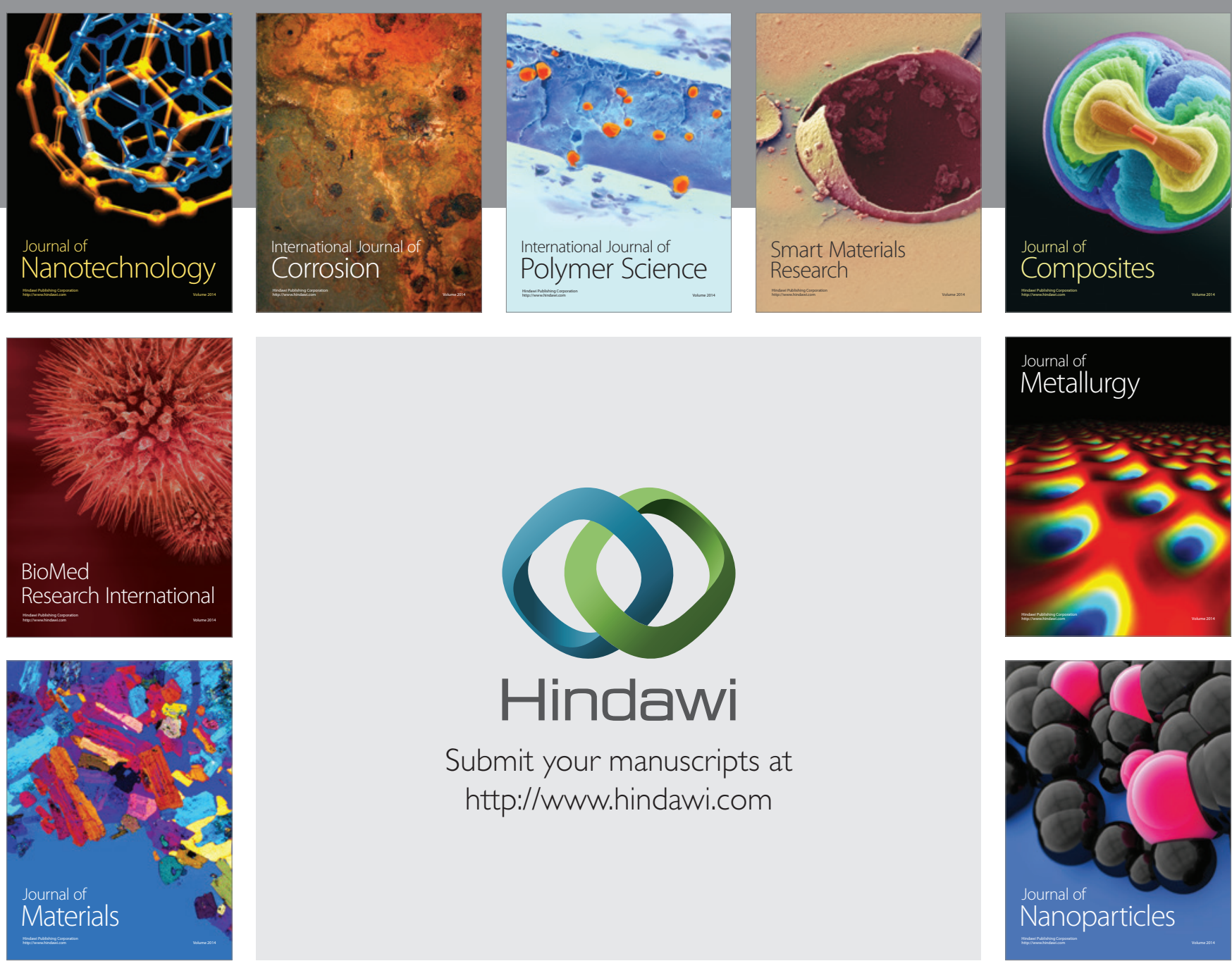

\section{Hindawi}

Submit your manuscripts at

http://www.hindawi.com

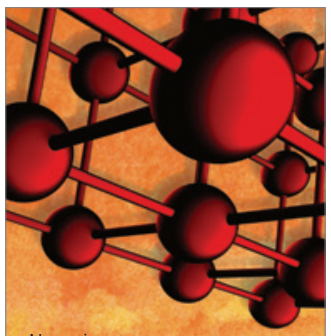

Materials Science and Engineering
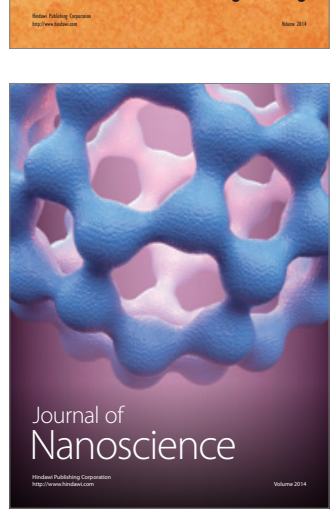
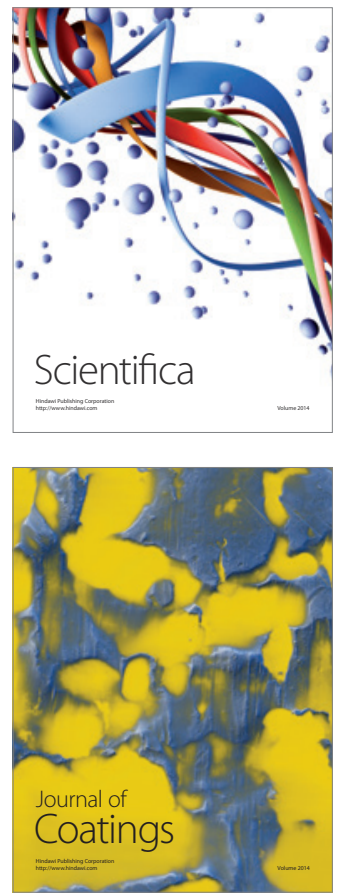
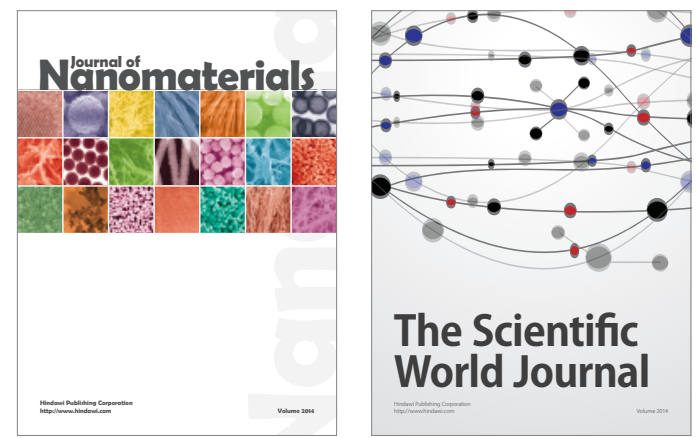

The Scientific World Journal
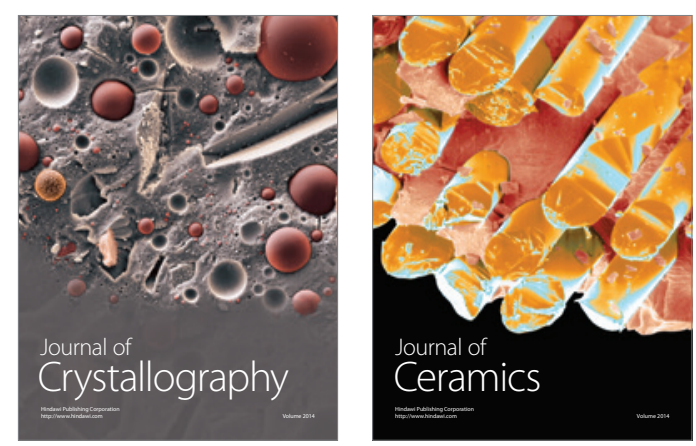
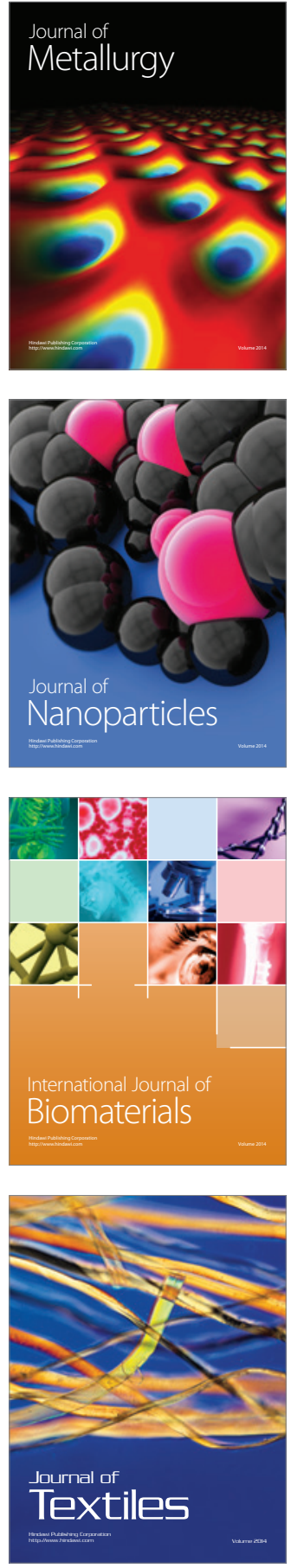